\title{
Improvement and generalization of some results related to the class of harmonically convex functions and applications
}

\author{
Imran Abbas Baloch ${ }^{\mathrm{a}, \mathrm{b}}$, Aqeel Ahmad Mughal ${ }^{\mathrm{c}}$, Yu Ming Chu ${ }^{\mathrm{d}, *}$, Absar Ul Haq ${ }^{e}$, Manuel De La Sen \\ a Abdus Salam School of Mathematical Sciences, GC University, Lahore, Pakistan. \\ ${ }^{b}$ Higher Education Department, Govt. College Gulberg, Punjab, Pakistan. \\ ${ }^{c}$ Department of Mathematics and Statistics, University of Lahore, Lahore, Pakistan. \\ ${ }^{d}$ Department of Mathematics, Huzhou University, Huzhou, China. \\ ${ }^{e}$ Department of Basic Sciences and Humanities, University of Engineering and Technology (Narowal Campus), Lahore 54000, Pakistan. \\ ${ }^{f}$ Institute of Research and Development of Processors, University of the Basque Country, campus of Leioa (Bizkaia), 48940 Leioa, \\ Spain.
}

\begin{abstract}
Discrete Jensen-type inequality for a harmonically convex function was established by Dragomir in [S. S. Dragomir, RGMIA Monographs, Victoria University, (2015)]. In [I. A. Baloch, A. H. Mughal, Y. M. Chu, M. De la Sen, Accepted in Aims Mathematics], Baloch et al. presented a variant of discrete Jensen-type inequality for harmonically convex functions. Moreover, they established a Jensen-type inequality for harmonically h-convex functions, and then they proved the variant of Jensen-type inequality for harmonically $h$-convex functions. Our results generalize and improve some earlier results in the literature (for example see [S. S. Dragomir, RGMIA Monographs, Victoria University, (2015)] and [I. A. Baloch, A. H. Mughal, Y. M. Chu, M. De la Sen, Accepted in Aims Mathematics]) for the said class. Additionally, using them gives us more interesting results.
\end{abstract}

Keywords: Harmonically convex functions, harmonically h-convex functions, Jensen-type inequality, a variant of Jensen-type inequality, harmonic series, HM-GM-AM inequality, a variant of HM-GM-AM.

2020 MSC: 26D15, 26A51, 26D10, 26A15.

(C)2021 All rights reserved.

\section{Introduction}

The convexity of functions has been frequently used in various fields of pure and applied mathematics, for instance in function theory, mathematical analysis, functional analysis, probability theory, optimization theory, operational research and information theory. In short, convex functions entail a strong and elegant interaction between analysis and geometry. The simple generalization to a convex function extensively widens our scope for analysis. Inequalities are frequently used in solving several problems of the applied sciences. Some recent work on the applications of mathematical inequalities can be found in $[1-8,15,16$, $18,19,22,23,25,27,28,30-32,34,36]$.

Jensen's inequality [24] is lord among inequalities because it make out at once the main part of the other classical inequalities (e.g. those by Hölder, Minkowski, Beckenbach-Dresher and Young, the A-G inequality etc.) that holds for the class of convex functions under certain conditions. In 2003, Mercer [26]

\footnotetext{
*Corresponding author

Email address: chuyuming2005@126. com (Yu Ming Chu)

doi: $10.22436 /$ jmcs.022.03.07
}

Received: 2020-03-31 Revised: 2020-05-07 Accepted: 2020-06-21 
gave a variant of Jensen's inequality which has an huge influence on the theory of inequalities. During the investigation of convexity, many researchers founded new classes of functions which are not convex in general. Some of them are the so called harmonically convex functions [21], harmonically $(\alpha, \mathrm{m})$-convex functions [20], harmonically $(s, m)$-convex functions $[11,13]$ and harmonically $(p,(s, m))$-convex functions [12]. For a quick glance on importance of these classes and applications see [10] and references therein.

Definition 1.1. A function $\mathrm{f}: \mathrm{I} \subseteq \mathbb{R} \backslash\{0\} \rightarrow \mathbb{R}$ is said to be harmonically convex function on I if

$$
f\left(\frac{x y}{t x+(1-t) y}\right) \leqslant t f(y)+(1-t) f(x)
$$

holds for all $x, y \in I$ and $t \in[0,1]$. If the inequality is reversed, then $f$ is said to be harmonically concave. In [10], Baloch et al. observed that class of harmonically convex functions is neither exactly that of convex functions nor it is entirely different from class of convex functions. Furthermore, they investigated remarkable relations between these two classes under certain conditions as follows.

Lemma 1.2. Let $\mathrm{I} \subseteq \mathbb{R} /\{0\}$ be a real interval. Define $\mathrm{I}^{-1}=\left\{\mathrm{y} \in \overline{\mathbb{R}}, \mathrm{y}=\frac{1}{x}, x \in \mathrm{I}\right\}$. A function $\mathrm{f}: \mathrm{I} \rightarrow \mathbb{R}$ is harmonically convex if and only if $\mathrm{g}: \mathrm{I}^{-1} \rightarrow \mathbb{R}$ is convex, where $\mathrm{g}$ is defined as $\mathrm{g}(\mathrm{y})=\mathrm{f}\left(\frac{1}{\mathrm{y}}\right)$.

Lemma 1.3. Let $\mathrm{I} \subseteq(0, \infty)$ and $\mathrm{I}^{-1}$ has similar definition as given in Lemma 1.2. A function $\mathrm{f}: \mathrm{I} \rightarrow \mathbb{R}$ is harmonically convex if and only if $\mathrm{h}: \mathrm{I} \rightarrow \mathbb{R}$ is convex, where $\mathrm{h}$ is defined as $\mathrm{h}(z)=z \mathrm{f}(\mathrm{z})$.

Proposition 1.4. Let $\mathrm{I} \subseteq \mathbb{R} /\{0\}$ be a real interval and $\mathrm{f}: \mathrm{I} \rightarrow \mathbb{R}$ is a function, then

1. if $\mathrm{I} \subset(0, \infty)$ and $\mathrm{f}$ is convex and nondecreasing function, then $\mathrm{f}$ is harmonically convex function;

2. if $\mathrm{I} \subset(0, \infty)$ and $\mathrm{f}$ is harmonically convex and nonincreasing function, then $\mathrm{f}$ is convex function;

3. if $\mathrm{I} \subset(-\infty, 0)$ and $\mathrm{f}$ is harmonically convex and nondecreasing function, then $\mathrm{f}$ is convex function;

4. if $\mathrm{I} \subset(-\infty, 0)$ and $\mathrm{f}$ is convex and nonincreasing function, then $\mathrm{f}$ is harmonically convex function.

Here, we reproduce some of the examples from article [10] for better understanding of our ongoing work. Now, first of all, we give an example of harmonically convex function which is not a convex function in Figure 1.

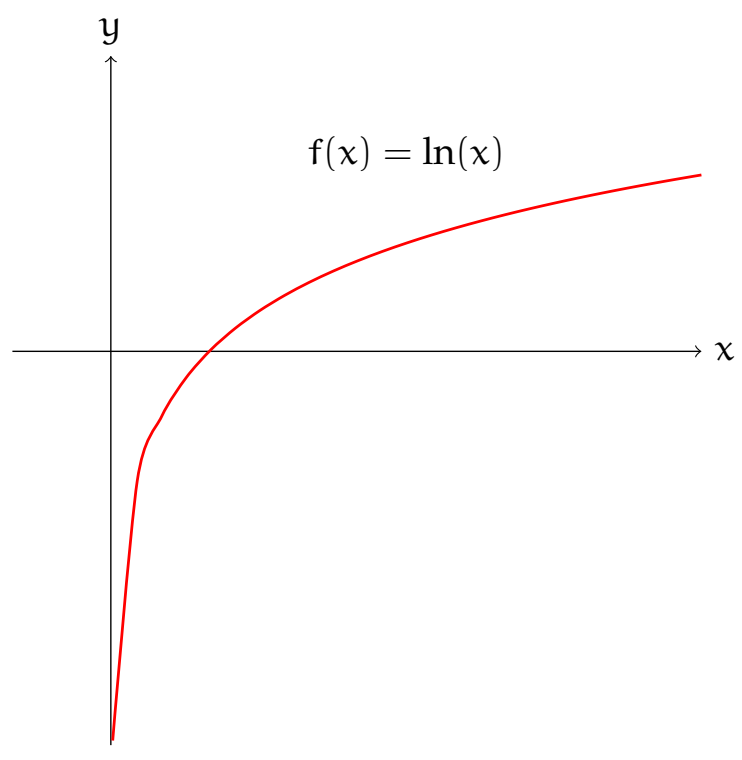

Figure 1

Here, we consider some more examples of harmonically convex functions in Figures 2 and 3. 


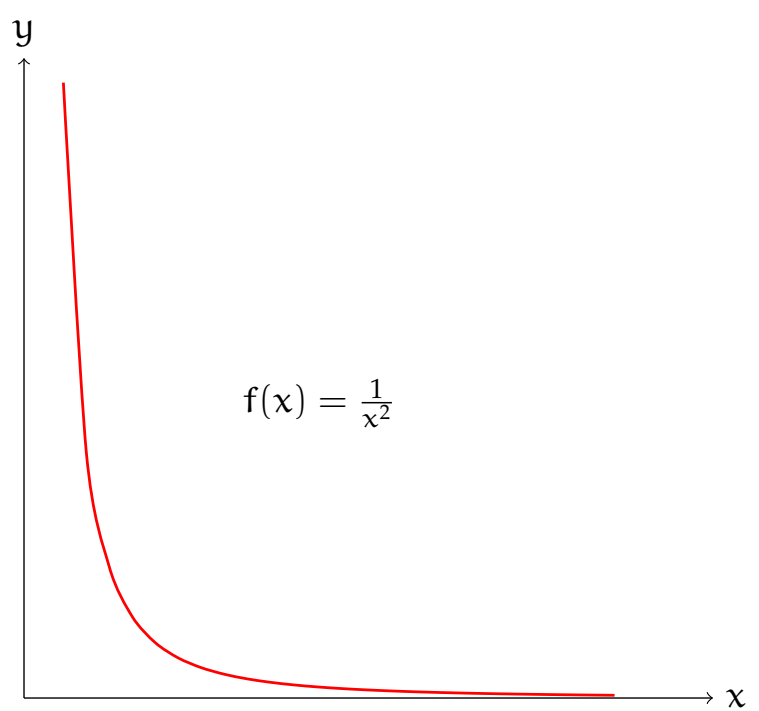

Figure 2

The above function is an example that these two classes have a non-empty intersection.
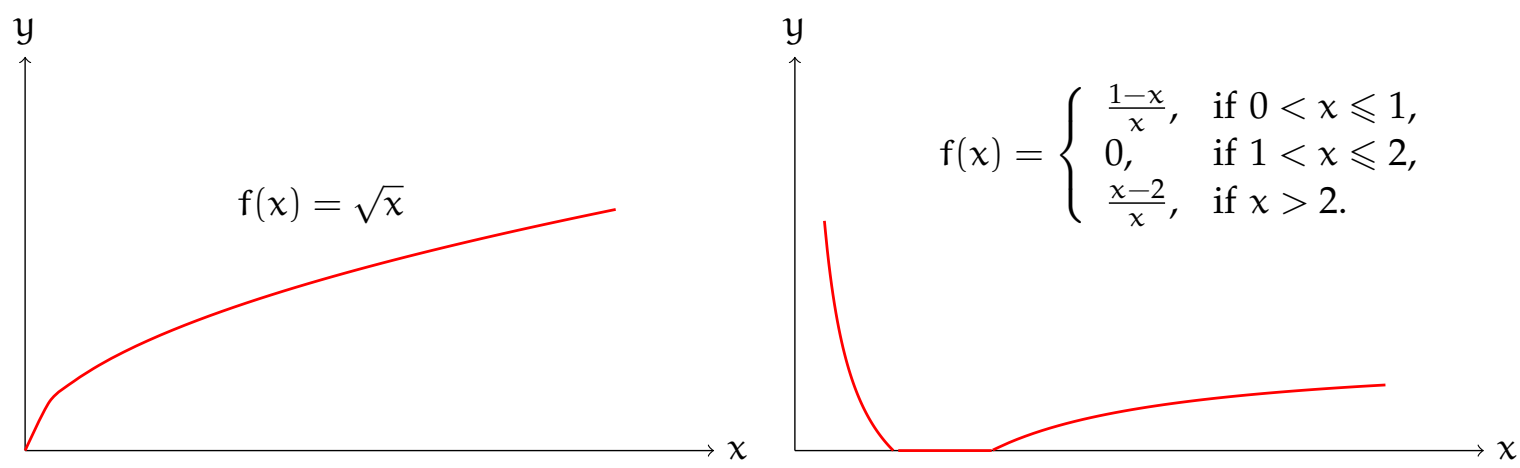

Figure 3

As h-convex functions which unify many other generalized aspects of convex functions, like s-convex functions, Godunova-Levin functions, s-Godunova-Levin functions and P-convex functions, was introduced by Varosanec in [35]. In a similar manner, class of harmonically h-convex was introduced to unify the various classes of harmonically convex functions. Here, we recall the definition of harmonically h-convex functions.

Definition 1.5 ([29]). Let $h:[0,1] \rightarrow \mathbb{R}$ be a non-negative function. A function $f: I \subseteq \mathbb{R} \backslash\{0\} \rightarrow \mathbb{R}$ is said to be harmonically $h$-convex function on $I$ if

$$
f\left(\frac{x y}{t x+(1-t) y}\right) \leqslant h(t) f(y)+h(1-t) f(x)
$$

holds for all $x, y \in I$ and $t \in[0,1]$. If the inequality is reversed, then $f$ is said to be harmonically concave.

In [17], Dragomir proved the following result, which is known as Jensen-type inequality for harmonically convex functions.

Theorem 1.6. Let $\mathrm{I} \subseteq(0, \infty)$ be an interval. If $\mathrm{f}: \mathrm{I} \rightarrow \mathbb{R}$ is harmonically convex function, then

$$
f\left(\frac{1}{\sum_{k=1}^{n} \frac{w_{k}}{x_{k}}}\right) \leqslant \sum_{k=1}^{n} w_{k} f\left(x_{k}\right),
$$


holds for all $x_{1}, \ldots, x_{n} \in \mathrm{I}$ and $w_{k} \in[0,1]$ with $\sum_{k=1}^{n} w_{k}=1$.

In [14], Baloch et al. proved the subsequent result.

Theorem 1.7. Let $\mathrm{I} \subseteq \mathbb{R} \backslash\{0\}$ be an interval. If $\mathrm{f}: \mathrm{I} \rightarrow \mathbb{R}$ is harmonically convex function, then for any finite positive sequence $\left(x_{k}\right)_{k=1}^{n} \in I$ and $w_{k} \in[0,1]$ with $\sum_{k=1}^{n} w_{k}=1$, we have

$$
f\left(\frac{1}{\frac{1}{x_{1}}+\frac{1}{x_{n}}-\sum_{k=1}^{n} \frac{w_{k}}{x_{k}}}\right) \leqslant f\left(x_{1}\right)+f\left(x_{n}\right)-\sum_{k=1}^{n} w_{k} f\left(x_{k}\right) .
$$

Also in [14], they corroborated Jensen-type inequality for harmonically h-convex function.

Theorem 1.8. Let $w_{1}, \ldots, w_{n}$ be positive real numbers $(n \geqslant 2)$ such that $w_{k} \in[0,1]$ and $\sum_{k=1}^{n} w_{k}=1$. If $\mathrm{h}: \mathrm{J} \supseteq(0,1) \rightarrow \mathbb{R}$ is a non-negative supermultiplicative function and if $\mathrm{f}$ is harmonically $\mathrm{h}$-convex function, then

$$
f\left(\frac{1}{\sum_{k=1}^{n} \frac{w_{k}}{x_{k}}}\right) \leqslant \sum_{k=1}^{n} h\left(w_{k}\right) f\left(x_{k}\right)
$$

holds for any finite positive sequence $\left(x_{\mathrm{k}}\right)_{\mathrm{k}=1}^{\mathrm{n}} \in \mathrm{I}$.

Further in [14], they proved a variant of Jensen-type inequality for harmonically h-convex function.

Theorem 1.9. Let $\mathrm{h}: \mathrm{J} \supseteq(0,1) \rightarrow \mathbb{R}$ be a non-negative supermutiplicative function on $\mathrm{J}$. Let $w_{1}, \ldots, w_{\mathrm{n}}$ be positive real numbers $(\mathrm{n} \geqslant 2)$ such that $\sum_{k=1}^{n} w_{k}=1$ and $\sum_{k=1}^{n} h\left(w_{k}\right) \leqslant 1$. If $f$ is a harmonically h-convex on $\mathrm{I} \subseteq \mathbb{R} \backslash\{0\}$, then for any finite positive increasing sequence $\left(\mathrm{x}_{\mathrm{k}}\right)_{\mathrm{k}=1}^{\mathrm{n}} \in \mathrm{I}$, we have

$$
f\left(\frac{1}{\frac{1}{x_{1}}+\frac{1}{x_{n}}-\sum_{k=1}^{n} \frac{w_{k}}{x_{k}}}\right) \leqslant f\left(x_{1}\right)+f\left(x_{n}\right)-\sum_{k=1}^{n} h\left(w_{k}\right) f\left(x_{k}\right) .
$$

If $\mathrm{h}$ is a submultiplicative function, $\sum_{\mathrm{k}=1}^{\mathrm{n}} \mathrm{h}\left(\boldsymbol{w}_{\mathrm{k}}\right) \geqslant 1$ and $\mathrm{f}$ is harmonically $\mathrm{h}$-concave then the inequality (1.4) is reversed.

Theorem 1.10 ([14]). Let $\mathrm{f}$ be a harmonically convex function on $[\mathrm{m}, \mathrm{M}]$. Then

$$
f\left(\frac{1}{\frac{1}{m}+\frac{1}{M}-\frac{a+b}{2 a b}}\right) \leqslant f(m)+f(M)-\int_{0}^{1} f\left(\frac{a b}{t a+(1-t) b}\right) d t \leqslant f(m)+f(M)-f\left(\frac{2 a b}{a+b}\right)
$$

for all $\mathrm{a}, \mathrm{b} \in[\mathrm{m}, \mathrm{M}]$.

In the next part, our main aim is to prove generalization and refinement of inequality (1.5) along with some particular inequalities. Further, a simple proof of weighted HGA inequality by use of our previous results for harmonically convex functions.

\section{Main results}

First of all, we establish a result similar to Theorem 1.10 under the same conditions.

Theorem 2.1. Let $\mathrm{f}$ be harmonically convex function on $[\mathrm{m}, \mathrm{M}]$. Then

$$
f\left(\frac{1}{\frac{1}{M}+\frac{1}{m}-\frac{a+b}{2 a b}}\right) \leqslant \frac{a b}{b-a} \int_{a}^{b} \frac{1}{t^{2}} f\left(\frac{1}{\frac{1}{m}+\frac{1}{M}-\frac{1}{t}}\right) d t \leqslant f(m)+f(M)-\frac{f(a)+f(b)}{2}
$$

for all $\mathrm{a}, \mathrm{b} \in[\mathrm{m}, \mathrm{M}]$. 
Proof. Since $\mathrm{f}$ is harmonically convex on $[\mathrm{m}, \mathrm{M}] \subset \mathbb{R} \backslash\{0\}$, then by the Hermite-Hadamard type inequality (2.2) of Theorem 2.4 from [21], we infer that

$$
\begin{aligned}
\int_{0}^{1} f\left(\frac{1}{\frac{1}{M}+\frac{1}{m}-\left(\frac{t}{a}+\frac{1-t}{b}\right)}\right) d t & =\int_{0}^{1} f\left(\frac{1}{t\left(\frac{1}{M}+\frac{1}{m}-\frac{1}{a}\right)+(1-t)\left(\frac{1}{M}+\frac{1}{m}-\frac{1}{b}\right)}\right) d t \\
& \geqslant f\left(\frac{2}{\left(\frac{1}{M}+\frac{1}{m}-\frac{1}{a}\right)+\left(\frac{1}{M}+\frac{1}{m}-\frac{1}{b}\right)}\right)=f\left(\frac{1}{\frac{1}{M}+\frac{1}{m}-\frac{a+b}{2 a b}}\right) .
\end{aligned}
$$

On the other hand, a variant of Jensen-type inequality (1.3) gives

$$
f\left(\frac{1}{\frac{1}{M}+\frac{1}{m}-\left(\frac{t}{a}+\frac{1-t}{b}\right)}\right) \leqslant f(M)+f(m)-(t f(a)+(1-t) f(b)) .
$$

Integrating both sides of (2.3), we get

$$
\begin{aligned}
\int_{0}^{1} f\left(\frac{1}{\frac{1}{M}+\frac{1}{m}-\left(\frac{t}{a}+\frac{1-t}{b}\right)}\right) d t & \leqslant f(M)+f(m)-\int_{0}^{1}(t f(a)+(1-t) f(b)) d t \\
& =f(M)+f(m)-\frac{f(a)+f(b)}{2}
\end{aligned}
$$

also

$$
\int_{0}^{1} f\left(\frac{1}{\frac{1}{M}+\frac{1}{m}-\left(\frac{t}{a}+\frac{1-t}{b}\right)}\right) d t=\frac{a b}{b-a} \int_{a}^{b} \frac{1}{t^{2}} f\left(\frac{1}{\frac{1}{m}+\frac{1}{M}-\frac{1}{t}}\right) d t .
$$

Hence, inequality (2.1) follows from inequalities (2.2), (2.4), and (2.5), this proves the assertion.

The following result has been proved in Lemma 2.1 by Baloch et al. [14], however we prove it here for the reader convenience but in a different way.

Lemma 2.2. Let $\mathrm{f}$ be harmonically convex function on $[\mathrm{m}, \mathrm{M}] \subset \mathbb{R} \backslash\{0\}$, then

$$
f\left(\frac{1}{\frac{1}{M}+\frac{1}{m}-\frac{1}{a_{k}}}\right) \leqslant f(M)+f(m)-f\left(a_{k}\right), \quad\left(m \leqslant a_{k} \leqslant M, k=1, \ldots, n\right) .
$$

Proof. If $\mathrm{f}:[\mathrm{m}, \mathrm{M}] \rightarrow \mathbb{R}$ is harmonically convex function, then for any $x, y \in[m, M]$ and $t \in[0,1]$, we have (1.1).

It can be verified that if $m \leqslant a_{k} \leqslant M(k=1, \ldots, n)$, then $\frac{m M-M a_{k}}{m a_{k}-M a_{k}}, \frac{m a_{k}-m M}{m a_{k}-M a_{k}} \leqslant 1$ and $\frac{m M-M a_{k}}{m a_{k}-M a_{k}}+$ $\frac{m a_{k}-m M}{m a_{k}-M a_{k}}=1$. Hence, by the use of (1.1), we have

$$
f\left(a_{k}\right) \leqslant \frac{m a_{k}-m M}{m a_{k}-M a_{k}} f(m)+\frac{m M-M a_{k}}{m a_{k}-M a_{k}} f(M)
$$

On the other hand, $m \leqslant a_{k} \leqslant M(k=1, \ldots, n)$ implies $m \leqslant \frac{1}{\frac{1}{M}+\frac{1}{m}-\frac{1}{a_{k}}} \leqslant M(k=1, \ldots, n)$. Thus, from (2.6), we conclude

$$
f\left(\frac{1}{\frac{1}{M}+\frac{1}{m}-\frac{1}{a_{k}}}\right) \leqslant \frac{m M-M a_{k}}{m a_{k}-M a_{k}} f(m)+\frac{m a_{k}-m M}{m a_{k}-M a_{k}} f(M) .
$$

Summing up (2.6) and (2.7), we get the desired result. 
Based on the above lemma, our first result can be stated as follows.

Theorem 2.3. Let $\mathrm{f}$ be a harmonically convex function on $[\mathrm{m}, \mathrm{M}] \subset \mathbb{R} \backslash\{0\}$. Then

$$
\begin{aligned}
f\left(\frac{1}{\frac{1}{M}+\frac{1}{m}-\sum_{k=1}^{n} \frac{w_{k}}{a_{k}}}\right) & \leqslant \sum_{k=1}^{n} w_{k} f\left(\frac{1}{\frac{1}{M}+\frac{1}{m}-\left(\sum_{k=1}^{n} w_{k} \frac{1-t}{a_{k}}+\frac{t}{a_{k}}\right)}\right) \\
& \leqslant f(M)+f(m)-\sum_{k=1}^{n} w_{k} f\left(a_{k}\right)
\end{aligned}
$$

for all $\mathrm{a}_{\mathrm{k}} \in[\mathrm{m}, \mathrm{M}]$ and $w_{\mathrm{k}} \in[0,1](\mathrm{k}=1, \ldots, \mathrm{n})$ with $\sum_{\mathrm{k}=1}^{\mathrm{n}} \boldsymbol{w}_{\mathrm{k}}=1$.

Proof. Firstly, since $f$ is harmonically convex function, we have

$$
\begin{aligned}
\sum_{k=1}^{n} w_{k} f\left(\frac{1}{\frac{1}{M}+\frac{1}{m}-\left(\sum_{k=1}^{n} w_{k} \frac{1-t}{a_{k}}+\frac{t}{a_{k}}\right)}\right) & \geqslant f\left(\frac{1}{\sum_{k=1}^{n} w_{k}\left(\frac{1}{M}+\frac{1}{m}-\left(\sum_{k=1}^{n} w_{k} \frac{1-t}{a_{k}}+\frac{t}{a_{k}}\right)\right)}\right) \\
& =f\left(\frac{1}{\frac{1}{M}+\frac{1}{m}-\sum_{k=1}^{n} \frac{w_{k}}{a_{k}}}\right) .
\end{aligned}
$$

On the other hand

$$
\begin{aligned}
\sum_{k=1}^{n} & w_{k} f\left(\frac{1}{\frac{1}{M}+\frac{1}{m}-\left(\sum_{k=1}^{n} w_{k} \frac{1-t}{a_{k}}+\frac{t}{a_{k}}\right)}\right) \\
& =\sum_{k=1}^{n} w_{k} f\left(\frac{1}{(1-t)\left(\frac{1}{M}+\frac{1}{m}-\sum_{k=1}^{n} \frac{w_{k}}{a_{k}}\right)+t\left(\frac{1}{M}+\frac{1}{m}-\frac{1}{a_{k}}\right)}\right) \\
& \leqslant \sum_{k=1}^{n} w_{k}\left[(1-t) f\left(\frac{1}{\frac{1}{M}+\frac{1}{m}-\sum_{k=1}^{n} \frac{w_{k}}{a_{k}}}\right)+t f\left(\frac{1}{\frac{1}{M}+\frac{1}{m}-\frac{1}{a_{k}}}\right)\right] \\
& =\sum_{k=1}^{n} w_{k}\left[(1-t)\left(f(M)+f(m)-\sum_{k=1}^{n} w_{k} f\left(a_{k}\right)\right)+t\left(f(M)+f(m)-f\left(a_{k}\right)\right)\right] \\
& =f(M)+f(m)-\sum_{k=1}^{n} w_{k} f\left(a_{k}\right) .
\end{aligned}
$$

Combining inequalities (2.9) and (2.10), we get (2.8). This proves the assertion.

Corollary 2.4. Let all assumptions of Theorem 2.3 hold, then

$$
\begin{aligned}
& f\left(\frac{1}{\frac{1}{M}+\frac{1}{m}-\sum_{k=1}^{n} \frac{w_{k}}{a_{k}}}\right) \\
& \leqslant \sum_{k=1}^{n} \frac{w_{k}}{\frac{1}{a_{k}}-\sum_{k=1}^{n} \frac{w_{k}}{a_{k}}} \int_{\frac{1}{M}+\frac{1}{m}-\sum_{k=1}^{n} \frac{w_{k}}{a_{k}}}^{\frac{1}{a_{k}}}\left[\frac{1}{\frac{1}{M}+\frac{1}{m}-\left(\sum_{k=1}^{n} w_{k} \frac{1-t}{a_{k}}+\frac{t}{a_{k}}\right)}\right]^{2} f(t) d t \\
& \leqslant f(M)+f(m)-\sum_{k=1}^{n} w_{k} f\left(a_{k}\right) \text {. }
\end{aligned}
$$

Proof. Integrating the inequality (2.8) over $t \in[0,1]$, we get (2.11). Here, we used the fact that

$$
\int_{0}^{1} f\left(\frac{1}{\frac{1}{M}+\frac{1}{m}-\left(\sum_{k=1}^{n} w_{k} \frac{1-t}{a_{k}}+\frac{t}{a_{k}}\right)}\right) d t
$$




$$
\begin{aligned}
& =\int_{0}^{1} f\left(\frac{1}{(1-t)\left(\frac{1}{M}+\frac{1}{m}-\sum_{k=1}^{n} \frac{w_{k}}{a_{k}}\right)+t\left(\frac{1}{M}+\frac{1}{m}-\frac{1}{a_{k}}\right)}\right) d t \\
& =\int_{0}^{1} f\left(\frac{1}{t\left(\frac{1}{M}+\frac{1}{m}-\sum_{k=1}^{n} \frac{w_{k}}{a_{k}}\right)+(1-t)\left(\frac{1}{M}+\frac{1}{m}-\frac{1}{a_{k}}\right)}\right) d t \\
& =\frac{1}{\frac{1}{a_{k}}-\sum_{k=1}^{n} \frac{w_{k}}{a_{k}}} \int_{\frac{1}{M}+\frac{1}{m}-\sum_{k=1}^{n} \frac{w_{k}}{a_{k}}}^{\frac{1}{M}+\frac{1}{m}-\frac{1}{a_{k}}}\left[\frac{1}{\frac{1}{M}+\frac{1}{m}-\left(\sum_{k=1}^{n} w_{k} \frac{1-t}{a_{k}}+\frac{t}{a_{k}}\right)}\right]^{2} f(t) \text {. }
\end{aligned}
$$

Remark 2.5. Put $\mathrm{n}=2, w_{1}=w_{2}=\frac{1}{2}, \mathrm{a}_{1}=\mathrm{a}$, and $\mathrm{a}_{2}=\mathrm{b}$ in Corollary 2.4, then we get

$$
f\left(\frac{1}{\frac{1}{M}+\frac{1}{m}-\frac{a+b}{2 a b}}\right) \leqslant \frac{a b}{b-a} \int_{a}^{b} \frac{1}{t^{2}} f\left(\frac{1}{\frac{1}{m}+\frac{1}{M}-\frac{1}{t}}\right) d t \leqslant f(m)+f(M)-\frac{f(a)+f(b)}{2},
$$

which declares that our inequality (2.11) generalizes our result in inequality (2.1).

Now, we present more precise estimate in the following theorem.

Theorem 2.6. Let $\mathrm{f}:[\mathrm{m}, \mathrm{M}] \subset \mathbb{R} \backslash\{0\} \rightarrow \mathbb{R}$ be a harmonically convex function. Then

$$
\begin{aligned}
& f\left(\frac{1}{\frac{1}{M}+\frac{1}{m}-\sum_{k=1}^{n} \frac{w_{k}}{a_{k}}}\right) \leqslant \sum_{k=1}^{n} w_{k} f\left(\frac{1}{\frac{1}{M}+\frac{1}{m}-\frac{1}{2}\left(\sum_{k=1}^{n} \frac{w_{k}}{a_{k}}+\frac{1}{a_{k}}\right)}\right) \\
& \leqslant \sum_{k=1}^{n} \frac{w_{k}}{\frac{1}{a_{k}}-\sum_{k=1}^{n} \frac{w_{k}}{a_{k}}} \int_{\frac{1}{M}+\frac{1}{m}-\sum_{k=1}^{n} \frac{w_{k}}{a_{k}}}^{\frac{1}{M}+\frac{1}{m}-\frac{1}{a_{k}}}\left[\frac{1}{\frac{1}{M}+\frac{1}{m}-\left(\sum_{k=1}^{n} w_{k} \frac{1-t}{a_{k}}+\frac{t}{a_{k}}\right)}\right]^{2} f(t) d t \\
& \leqslant f(M)+f(m)-\sum_{k=1}^{n} w_{k} f\left(a_{k}\right)
\end{aligned}
$$

for all $\mathrm{a}_{\mathrm{k}} \in[\mathrm{m}, \mathrm{M}]$ and $w_{\mathrm{k}} \in[0,1](\mathrm{k}=1, \ldots, \mathrm{n})$ with $\sum_{\mathrm{k}=1}^{\mathrm{n}} \mathrm{w}_{\mathrm{k}}=1$.

Proof. If $f:[m, M] \subset \mathbb{R} \backslash\{0\} \rightarrow \mathbb{R}$ is a harmonically convex function, then, we have for any $a, b \in[m, M]$

$$
f\left(\frac{2 a b}{a+b}\right)=f\left(\frac{1}{\frac{1}{2}\left(\frac{t}{a}+\frac{1-t}{b}+\frac{1-t}{a}+\frac{t}{b}\right)}\right) \leqslant \frac{1}{2} f\left(\frac{1}{\frac{t}{a}+\frac{1-t}{b}}\right)+\frac{1}{2} f\left(\frac{1}{\frac{1-t}{a}+\frac{t}{b}}\right) \leqslant \frac{f(a)+f(b)}{2} .
$$

Replacing $a$ and $b$ by $\frac{1}{\frac{1}{M}+\frac{1}{m}-\frac{1}{a}}$ and $\frac{1}{\frac{1}{M}+\frac{1}{m}-\frac{1}{b}}$, respectively, we get

$$
\begin{aligned}
f\left(\frac{1}{\frac{1}{M}+\frac{1}{m}-\frac{a+b}{2 a b}}\right) & \leqslant \frac{1}{2}\left[f\left(\frac{1}{\frac{1}{M}+\frac{1}{m}-\left(\frac{t}{a}+\frac{1-t}{b}\right)}\right)+f\left(\frac{1}{\frac{1}{M}+\frac{1}{m}-\left(\frac{1-t}{a}+\frac{t}{b}\right)}\right)\right] \\
& \leqslant \frac{1}{2}\left[f\left(\frac{1}{\frac{1}{M}+\frac{1}{m}-\frac{1}{a}}\right)+f\left(\frac{1}{\frac{1}{M}+\frac{1}{m}-\frac{1}{b}}\right)\right] .
\end{aligned}
$$

Integrating the above inequality over $t \in[0,1]$, and using fact that

$$
\int_{0}^{1} f\left(\frac{1}{\frac{1}{M}+\frac{1}{m}-\left(\frac{t}{a}+\frac{1-t}{b}\right)}\right) d t=\int_{0}^{1} f\left(\frac{1}{\frac{1}{M}+\frac{1}{m}-\left(\frac{1-t}{a}+\frac{t}{b}\right)}\right) d t,
$$

we conclude that

$$
f\left(\frac{1}{\frac{1}{M}+\frac{1}{m}-\frac{a+b}{2 a b}}\right) \leqslant \int_{0}^{1} f\left(\frac{1}{\frac{1}{M}+\frac{1}{m}-\left(\frac{t}{a}+\frac{1-t}{b}\right)}\right) d t \leqslant \frac{1}{2}\left[f\left(\frac{1}{\frac{1}{M}+\frac{1}{m}-\frac{1}{a}}\right)+f\left(\frac{1}{\frac{1}{M}+\frac{1}{m}-\frac{1}{b}}\right)\right] .
$$


Since, $a_{k}, \sum_{k=1}^{n} \frac{w_{k}}{a_{k}} \in[m, M]$, hence we can write

$$
\begin{aligned}
f\left(\frac{1}{\frac{1}{M}+\frac{1}{m}-\frac{1}{2}\left(\sum_{k=1}^{n} \frac{w_{k}}{a_{k}}+\frac{1}{a_{k}}\right)}\right) & \leqslant \frac{1}{\frac{1}{a_{k}}-\sum_{k=1}^{n} \frac{w_{k}}{a_{k}}} \int_{\frac{1}{M}+\frac{1}{m}-\frac{1}{a_{k}}}^{\frac{1}{M}+\frac{1}{m}-\sum_{k=1}^{n} \frac{w_{k}}{a_{k}}}\left[\frac{1}{\frac{1}{M}+\frac{1}{m}-\left(\sum_{k=1}^{n} w_{k} \frac{1-t}{a_{k}}+\frac{t}{a_{k}}\right)}\right]^{2} f(t) d t \\
& \leqslant \frac{1}{2}\left[f\left(\frac{1}{\frac{1}{M}+\frac{1}{m}-\sum_{k=1}^{n} \frac{w_{k}}{a_{k}}}\right)+f\left(\frac{1}{\frac{1}{M}+\frac{1}{m}-\frac{1}{a_{k}}}\right)\right],
\end{aligned}
$$

where

$$
\begin{aligned}
& \int_{0}^{1} f\left(\frac{1}{\frac{1}{M}+\frac{1}{m}-\left(\sum_{k=1}^{n} \frac{t w_{k}}{a_{k}}+\frac{1}{a_{k}}\right)}\right) d t \\
& =\frac{1}{\frac{1}{a_{k}}-\sum_{k=1}^{n} \frac{w_{k}}{a_{k}}} \int_{\frac{1}{M}+\frac{1}{m}-\frac{1}{a_{k}}}^{\frac{1}{m}-\sum_{k=1}^{n} \frac{w_{k}}{a_{k}}}\left[\frac{1}{\frac{1}{M}+\frac{1}{m}-\left(\sum_{k=1}^{n} w_{k} \frac{1-t}{a_{k}}+\frac{t}{a_{k}}\right)}\right]^{2} f(t) d t .
\end{aligned}
$$

Multiplying by $w_{k}>0(k=1, \ldots, n)$ and summing over $k$ from 1 to $n$, we have

$$
\begin{aligned}
& \sum_{k=1}^{n} w_{k} f\left(\frac{1}{\frac{1}{M}+\frac{1}{m}-\frac{1}{2}\left(\sum_{k=1}^{n} \frac{w_{k}}{a_{k}}+\frac{1}{a_{k}}\right)}\right) \\
& \leqslant \sum_{k=1}^{n} \frac{w_{k}}{\frac{1}{a_{k}}-\sum_{k=1}^{n} \frac{w_{k}}{a_{k}}} \int_{\frac{1}{M}+\frac{1}{m}-\frac{1}{a_{k}}}^{\frac{1}{M}+\frac{1}{m}-\sum_{k=1}^{n} \frac{w_{k}}{a_{k}}}\left[\frac{1}{\frac{1}{M}+\frac{1}{m}-\left(\sum_{k=1}^{n} w_{k} \frac{1-t}{a_{k}}+\frac{t}{a_{k}}\right)}\right]^{2} f(t) d t \\
& \leqslant
\end{aligned}
$$

On the other hand, by (1.2)

$$
\begin{aligned}
& f\left(\frac{1}{\sum_{k=1}^{n} w_{k}\left(\frac{1}{M}+\frac{1}{m}-\sum_{k=1}^{n} \frac{w_{k}}{a_{k}}\right)}\right)=f\left(\frac{1}{\sum_{k=1}^{n} w_{k}\left(\frac{1}{M}+\frac{1}{m}-\frac{1}{2}\left(\sum_{k=1}^{n} \frac{w_{k}}{a_{k}}+\frac{1}{a_{k}}\right)\right)}\right) \\
& \quad \leqslant \sum_{k=1}^{n} w_{k} f\left(\frac{1}{\frac{1}{M}+\frac{1}{m}-\frac{1}{2}\left(\sum_{k=1}^{n} \frac{w_{k}}{a_{k}}+\frac{1}{a_{k}}\right)}\right)
\end{aligned}
$$

and by Theorem 1.7 and Lemma 2.2

$$
\begin{aligned}
& \frac{1}{2}\left[f\left(\frac{1}{\frac{1}{M}+\frac{1}{m}-\sum_{k=1}^{n} \frac{w_{k}}{a_{k}}}\right)+\sum_{k=1}^{n} f\left(\frac{1}{\frac{1}{M}+\frac{1}{m}-\frac{1}{a_{k}}}\right)\right] \\
& \quad \leqslant \frac{1}{2}\left[f(M)+f(m)-\sum_{k=1}^{n} w_{k} f\left(a_{k}\right)+f(M)+f(m)-\sum_{k=1}^{n} w_{k} f\left(a_{k}\right)\right]=f(M)+f(m)-\sum_{k=1}^{n} w_{k} f\left(a_{k}\right) .
\end{aligned}
$$

Combining (2.13), (2.14), and (2.15), we get (2.12), which proves the theorem. 
Corollary 2.7. Let $\mathrm{a}_{\mathrm{k}} \in[\mathrm{m}, \mathrm{M}]$ and $w_{\mathrm{k}} \in[0,1](\mathrm{k}=1, \ldots, \mathrm{n})$ with $\sum_{\mathrm{k}=1}^{\mathrm{n}} \mathrm{w}_{\mathrm{k}}=1$. Then

$$
\begin{aligned}
& \frac{1}{\frac{1}{M}+\frac{1}{m}-\sum_{k=1}^{n} \frac{w_{k}}{a_{k}}} \leqslant \prod_{k=1}^{n}\left(\frac{1}{\frac{1}{M}+\frac{1}{m}-\frac{1}{2}\left(\sum_{k=1}^{n} \frac{w_{k}}{a_{k}}+\frac{1}{a_{k}}\right)}\right)^{w_{k}} \\
& \leqslant \exp \left[\sum_{k=1}^{n} \frac{w_{k}}{\frac{1}{a_{k}}-\sum_{k=1}^{n} \frac{w_{k}}{a_{k}}}\right. \\
& \left.\times \int_{\frac{1}{M}+\frac{1}{m}-\sum_{k=1}^{n} \frac{w_{k}}{a_{k}}}^{\frac{1}{M}+\frac{1}{m}-\frac{1}{a_{k}}}\left[\frac{1}{\frac{1}{M}+\frac{1}{m}-\left(\sum_{k=1}^{n} w_{k} \frac{1-t}{a_{k}}+\frac{t}{a_{k}}\right)}\right]^{2} f(t) d t\right] \\
& \leqslant \frac{m M}{\prod_{k=1}^{n} a_{k}^{w_{k}}} \text {. }
\end{aligned}
$$

Proof. Put $f(t)=\log t,(0<t)$ in Theorem 2.6, we get the inequality (2.16) after some simplifications.

Remark 2.8. If we set $n=2, a_{1}=m, a_{2}=M$ and $w_{1}=w_{2}=\frac{1}{2}$ in Corollary 2.7, then we have

$$
\frac{2 m M}{m+M} \leqslant \frac{4 m M}{\sqrt{(3 m+M)(m+3 M)}} \leqslant A_{m, M} \leqslant \sqrt{m M}
$$

where

$$
A_{m, M}=\exp \left[\sum_{k=1}^{n} \frac{w_{k}}{\frac{1}{a_{k}}-\sum_{k=1}^{n} \frac{w_{k}}{a_{k}}} \int_{\frac{1}{M}+\frac{1}{m}-\sum_{k=1}^{n} \frac{w_{k}}{a_{k}}}^{\frac{1}{m}-\frac{1}{a_{k}}}\left[\frac{1}{\frac{1}{M}+\frac{1}{m}-\left(\sum_{k=1}^{n} w_{k} \frac{1-t}{a_{k}}+\frac{t}{a_{k}}\right)}\right]^{2} f(t) d t\right] .
$$

Remark 2.9.

$$
\begin{aligned}
f\left(\frac{1}{\sum_{k=1}^{n} \frac{w_{k}}{a_{k}}}\right) & \leqslant \sum_{k=1}^{n} w_{k} f\left(\frac{2}{\sum_{k=1}^{n} \frac{w_{k}}{a_{k}}+\frac{1}{a_{k}}}\right) \\
& \leqslant \sum_{k=1}^{n} \frac{w_{k}}{\sum_{k=1}^{n} \frac{w_{k}}{a_{k}}-\frac{1}{a_{k}}} \int_{\frac{1}{\sum_{k=1}^{n} \frac{w_{k}}{a_{k}}}}^{a_{k}}\left(\frac{1}{\sum_{k=1}^{n} w_{k} \frac{1-t}{a_{k}}+\frac{t}{a_{k}}}\right)^{2} f(t) d t \leqslant \sum_{k=1}^{n} w_{k} f\left(a_{k}\right) .
\end{aligned}
$$

\section{Applications}

In this section, we give interesting applications of our results. Since $f(x)=x, \forall x \in(0, \infty)$ is harmonically convex function, so from inequality (1.2) we get

$$
\frac{1}{\sum_{k=1}^{n} \frac{w_{k}}{x_{k}}} \leqslant \sum_{k=1}^{n} w_{k} x_{k}
$$

Taking

$$
x_{k}=k \text { with } w_{k}=\frac{1}{n}(1 \leqslant k \leqslant n)
$$

in inequality (3.1), we get

$$
\frac{2 n}{n+1} \leqslant \sum_{m=1}^{n} \frac{1}{m}
$$

Now, using inequality (1.3) for $f(x)=x, \forall x \in(0, \infty)$ we have

$$
\frac{1}{\frac{1}{x_{1}}+\frac{1}{x_{n}}-\sum_{k=1}^{n} \frac{w_{k}}{x_{k}}} \leqslant x_{1}+x_{n}-\sum_{k=1}^{n} w_{k} x_{k}
$$


and under condition (3.2), inequality (3.4) becomes

$$
\sum_{m=1}^{n} \frac{1}{m} \leqslant \frac{n^{2}+1}{n+1}
$$

Hence, by combining inequalities (3.3) and (3.5), we get

$$
\frac{2 n}{n+1} \leqslant \sum_{m=1}^{n} \frac{1}{m} \leqslant \frac{n^{2}+1}{n+1} .
$$

Next, since $f(x)=\ln x, \forall x \in(0, \infty)$ is harmonically convex function and concave function, so from inequality (1.2) and classical Jensen's inequality and variant of classical Jensen's inequality, we get

$$
\frac{1}{\sum_{k=1}^{n} \frac{w_{k}}{x_{k}}} \leqslant \prod_{k=1}^{n} x_{k}^{w_{k}}
$$

and

$$
\begin{gathered}
\prod_{k=1}^{n} x_{k}^{w_{k}} \leqslant \sum_{k=1}^{n} w_{k} x_{k}, \\
x_{1} x_{n} \prod_{k=1}^{n} x_{k}^{-w_{k}} \leqslant x_{1}+x_{n}-\sum_{k=1}^{n} w_{k} x_{k} .
\end{gathered}
$$

Now, using inequality (1.3) for $f(x)=\ln x, \forall x \in(0, \infty)$ we have

$$
\prod_{k=1}^{n} x_{k}^{w_{k}} \leqslant\left(x_{1}+x_{n}\right)-x_{1} x_{n} \sum_{k=1}^{n} \frac{w_{k}}{x_{k}}
$$

or

$$
\left(x_{1}^{-1}+x_{n}^{-1}-\sum_{k=1}^{n} w_{k} x_{k}^{-1}\right)^{-1} \leqslant x_{1} x_{n} \prod_{k=1}^{n} x_{k}^{-w_{k}} .
$$

Here, we conjecture a result and left its proof for researcher, as follows

$$
\sum_{k=1}^{n} w_{k} x_{k} \leqslant\left(x_{1}+x_{n}\right)-x_{1} x_{n} \sum_{k=1}^{n} \frac{w_{k}}{x_{k}}
$$

and hence, from inequalities (3.1), (3.7), (3.8), (3.10), and (3.12), we conclude that

$$
\frac{1}{\sum_{k=1}^{n} \frac{w_{k}}{x_{k}}} \leqslant \prod_{k=1}^{n} x_{k}^{w_{k}} \leqslant \sum_{k=1}^{n} w_{k} x_{k} \leqslant\left(x_{1}+x_{n}\right)-x_{1} x_{n} \sum_{k=1}^{n} \frac{w_{k}}{x_{k}} .
$$

However, if the conjecture (3.12) is not true, even then we have given the simple proof of

$$
\frac{1}{\sum_{k=1}^{n} \frac{w_{k}}{x_{k}}} \leqslant \prod_{k=1}^{n} x_{k}^{w_{k}} \leqslant \sum_{k=1}^{n} w_{k} x_{k} \text {, weighted HGA inequality. }
$$

In particularly, for $w_{k}$ as given in condition (3.2), the inequality (3.13) becomes

$$
\frac{n}{\frac{1}{x_{1}}+\frac{1}{x_{2}}+\cdots+\frac{1}{x_{n}}} \leqslant \sqrt[n]{x_{1} x_{2} \cdots x_{n}} \leqslant \frac{x_{n}+x_{n}+\cdots+x_{n}}{n}, \text { HGA inequality. }
$$


For $x_{k}=\frac{1}{k} 1 \leqslant k \leqslant n$, we get from (3.14),

$$
\frac{2 n}{n+1} \leqslant \frac{n}{\sqrt[n]{\prod_{k=1}^{n} k}} \leqslant \sum_{m=1}^{n} \frac{1}{m}
$$

Also from inequalities (3.9) and (3.11), we get

$$
\left(x_{1}^{-1}+x_{n}^{-1}-\sum_{k=1}^{n} w_{k} x_{k}^{-1}\right)^{-1} \leqslant x_{1} x_{n} \prod_{k=1}^{n} x_{k}^{-w_{k}} \leqslant x_{1}+x_{n}-\sum_{k=1}^{n} w_{k} x_{k} .
$$

Now, if $I=[a, b]$ and $x_{k} \in I,(1 \leqslant k \leqslant n)$ then by the virtue of inequality (1.3), the above inequality becomes

$$
\left(a^{-1}+b^{-1}-\sum_{k=1}^{n} w_{k} x_{k}^{-1}\right)^{-1} \leqslant a b \prod_{k=1}^{n} x_{k}^{-w_{k}} \leqslant a+b-\sum_{k=1}^{n} w_{k} x_{k},
$$

since the above inequality holds for arbitrary value of $n$, infinite convex combination $\sum_{k=1}^{\infty} w_{k} x_{k}$ converges in I and then affine combination $a+b-\sum_{k=1}^{\infty} w_{k} x_{k}$ converges in I, see for example [33], so it must be true as $n$ tends to $\infty$, i.e.,

$$
\left(a^{-1}+b^{-1}-\sum_{k=1}^{\infty} w_{k} x_{k}^{-1}\right)^{-1} \leqslant a b \prod_{k=1}^{\infty} x_{k}^{-w_{k}} \leqslant a+b-\sum_{k=1}^{\infty} w_{k} x_{k} .
$$

Now, first use (1.2) for harmonically convex function $f(x)=\frac{1}{x^{2}}$ and then by assuming condition (3.2), we have

$$
\sum_{m=1}^{n} \frac{1}{m} \leqslant \sqrt{n \sum_{m=1}^{n} \frac{1}{m^{2}}}
$$

By virtue of inequalities (3.15) and (3.16), we get a refinement of (3.6) as

$$
\frac{2 n}{n+1} \leqslant \frac{n}{\sqrt[n]{\prod_{k=1}^{n} k}} \leqslant \sum_{m=1}^{n} \frac{1}{m} \leqslant \sqrt{n \sum_{m=1}^{n} \frac{1}{m^{2}}} \leqslant n \sqrt{\frac{2}{n+1}} \leqslant \frac{n^{2}+1}{n+1} .
$$

\section{Conclusion}

The harmonically convexity of a function is the basis for many inequalities in mathematics as you may see in $([9,11-14,20,21])$ and references therein. Furthermore, harmonically convexity provides an analytic tool to estimate several known definite integrals like $\int_{a}^{b} \frac{e^{t}}{t^{n}} d t, \int_{a}^{b} e^{t^{2}} d t, \int_{a}^{b} \frac{\sin t}{t^{n}} d t$ and $\int_{a}^{b} \frac{\cos t}{t^{n}} d t$, $\forall \mathrm{n} \in \mathbb{N}$, where $\mathrm{a}, \mathrm{b} \in(0, \infty)$, see [10]. In this article, we have given the improvements and generalizations of some results from $[14,17]$ and moreover, we used these results for the particular functions to get the improvements of results already exist in literature. Further, we found sharp bounds for $\sum_{m=1}^{n} \frac{1}{m}$ and gave a simple proof of weighted HGA-means inequality. We hope techniques and consequences of this article will inspire the researcher to explore more interesting sequel in this area.

\section{Acknowledgment}

The authors are grateful to the Basque Government by its support through Grant IT1207/19. This research article is partially supported by Higher Education Commission of Pakistan too. 


\section{References}

[1] A. O. Akdemir, M. E. Özdemir, $(\mathrm{h}, \mathrm{m})$.convex functions on $\Delta=[\mathrm{a}, \mathrm{b}] \times[\mathrm{c}, \mathrm{d}]$ and Hadamard-type integral inequality, AIP Conference Proceedings, 1833 (2017), 20-28. 1

[2] A. O. Akdemir, M. E. Özdemir, M. Avcii-Ardiç, A. Yalçiin, Some new generalizations for GA-convex functions, Filomat, 31 (2017), 1009-1019.

[3] A. O. Akdemir, M. E. Özdemir, S. Varosanec, On some inequalities for h-concave functions, Math. Comput. Modelling, 55 (2012), 746-753.

[4] A. O. Akdemir, E. Set, M. E. Özdemir, C. Yiildiiz, On some new inequalities of Hadamard type for h-convex functions, American Inst. Phys., AIP Conference Proceedings, 1470 (2012), 35-38.

[5] A. O. Akdemir, A. Yalçıin, F. Polat, H. Kavurmaci-Önalan, Geometric-Harmonic convexity and integral inequalities, American Inst. Phys., (AIP) Conference Proceedings, 1726 (2016), 15 pages.

[6] G. D. Anderson, M. K. Vamanamurthy, M. Vuorinen, Generalized convexity and inequalities, J. Math. Anal. Appl., 335 (2007), 1294-1308.

[7] M. Avcii-Ardiç, A. O. Akdemir, E. Set, New Ostrowski Like Inequalities For GG-Convex and GA-Convex Functions, Math. Inequal. Appl., 19 (2016), 1159-1168.

[8] M. Avcii-Ardiç, A. O. Akdemir, K. Yuildiiz, On Some New Inequalities Via GG-Convexity And GA-Convexity, Filomat, 32 (2018), 5707-5717. 1

[9] I. A. Baloch, Y.-M. Chu, Petrović-type inequalities for harmonic h-convex functions, J. Funct. Space, 2020 (2020), 7 pages. 4

[10] I. A. Baloch, M. De la Sen, İ. İşcan, Characterizations of Classes of Harmonic Convex Functions and Applications, Int. J. Anal. Appl., 17 (2019), 722-733. 1, 1.1, 1.1, 4

[11] I. A. Baloch, İ. İşcan, Some Ostrowski type inequalities For harmonically (s, m)-convex functions in second sense, Int. J. Anal., 2015 (2015), 9 pages. 1, 4

[12] I. A. Baloch, İ. İşcan, Some Hermite-Hadamard type integral inequalities for Harmonically $(p,(s, m))$-convex functions, J. Inequal. Spec. Funct., 8 (2017), 65-84. 1

[13] I. A. Baloch, İ. İşcan, S. S. Dragomir, Fejér type inequalities for harmonically (s, m)-convex functions International Journal of Analysis and Applications, Int. J. Anal. Appl., 12 (2016), 188-197. 1

[14] I. A. Baloch, A. H. Mughal, Y. M. Chu, M. De la Sen, A variant of Jensen-type inequality and related results for harmonic convex functions, Accepted in Aims Mathematics. 1, 1, 1, 1.10, 2, 4

[15] M. Bombardelli, S. Varošanec, Properties of h-convex functions related to the Hermite Hadamard Fejér inequalities, Comput. Math. Appl., 58 (2009), 1869-1877. 1

[16] S. S. Dragomir, Bounds for the normalised Jensen functional, Bull. Aust. Math. Soc., 74 (2006), 471-478. 1

[17] S. S. Dragomir, Inequality of Jensen-type for HA-convex functions, RGMIA Monographs, Victoria University, (2015). 1,4

[18] L. Horváth, K. A. Khan, J. Pečarić, Cyclic refinements of the discrete and integral form of Jensen's inequality with applications, Analysis, 36 (2016), 253-262. 1

[19] L. Horváth, D. Pečarić, J. Pečarić, Estimations of f-and Rényi divergences by using a cyclic refinement of the Jensen's inequality, Bull. Malays. Math. Sci. Soc. (2), 42 (2019), 933-946. 1

[20] İ. İscan, Hermite-Hadamard type inequalities for harmonically $(\alpha, \mathrm{m})$-convex functions, arXiv, 2013 (2013), 10 pages. 1, 4

[21] İ. İşcan, Hermite-Hadamard type inequaities for harmonically convex functions, Hacet. J. Math. Stat., 43 (2014), $935-942$. $1,2,4$

[22] J. Jakšetić, J. Pečarić, Exponential convexity method, J. Convex Anal., 20 (2013), 181-197. 1

[23] J. Jakšetić, D. Pečarić, J. Pečarić, Some properties of Zipf-Mandelbrot law and Hurwitz-function, Math. Inequal. Appl., 21 (2018), 575-584. 1

[24] J. L. W. V. Jensen, Sur les fonctions convexes et inégalités entre les valeurs moyennes, Acta Math., 30 (1906), 175-193. 1

[25] N. Mehmood, S. I. Butt, D. Pečarić, J. Pečarić, Several new cyclic Jensen type inequalities and their applications, J. Inequal. Appl., 2019 (2019), 18 pages. 1

[26] A. M. Mercer, A variant of Jensen's inequality, JIPAM. J. Inequal. Pure Appl. Math., 4 (2003), 2 pages. 1

[27] İ. Mumcu, E. Set, A. O. Akdemir, Hermite-Hadamard type inequalities for harmonically convex functions via katugampola fractional integrals, Miskolc Math. Notes, 20 (2019), 409-424. 1

[28] C. P. Niculescu, L.-E. Persson, Convex functions and their applications, Springer-Verlag, New York, (2006). 1

[29] M. A. Noor, K. I. Noor, M. U. Awan, S. Costache, Some integral inequalities for harmonically h-convex functions, Politehn. Univ. Bucharest Sci. Bull. Ser. A Appl. Math. Phys., 77 (2015), 5-16. 1.5

[30] N. Okur, F. B. Yalçin, Two dimensional operator harmonically convex functions and related generalized inequalities, Turkish J. Sci., 4 (2019), 30-38. 1

[31] M. E. Özdemir, A. O. Akdemir, E. Set, On (h, m)-convexity and Hadamard-type inequalities, Transylv. J. Math. Mech., 8 (2016), 51-58.

[32] M. E. Özdemir, M. A. Latif, A. O. Akdemir, On some Hadamard-Type inequalities for product of two convex functions on the co-ordinates, Turkish J. Sci., 1 (2016), 41-58. 1

[33] Z. Pavic, The Jensen-Mercer inequality with infinite convex combinations, Math. Sci. Appl. E-Notes, 7 (2019), 19-27. 3 
[34] J. E. Pečarić, F. Proschan, Y. L. Tong, Convex functions, partial orderings and statistical applications, Academic Press, Boston, (1992). 1

[35] S. Varošanec, On h-convexity, J. Math. Anal. Appl., 326 (2007), 303-311. 1

[36] J. C. Zhao, S. I. Butt, J. Nasir, Z. B. Wang, I. Tlili, Hermite Jensen Mercer Type Inequalities for Caputo Fractional Derivatives, J. Funct. Spaces, 2020 (2020), 11 pages. 1 